\title{
Application of a quantitative PCR assay to evaluate Human Epidermal Growth Factor Receptor - 2 status of Gastric carcinoma in a cohort of Sri Lankan patients
}

\author{
D. K. S. Kannangara ${ }^{1}$, D. Subasinghe ${ }^{2}$, M. D. S. Lokuhetty ${ }^{3}$, R. S. Dassanayake ${ }^{4}$ and \\ Y. I. N. S. Gunawardene ${ }^{1}$ \\ Molecular Medicine Unit, Faculty of Medicine, University of Kelaniya, Sri Lanka ${ }^{l}$ \\ Professorial Surgical Unit, National Hospital of Sri Lanka ${ }^{2}$ \\ Department of Pathology, Faculty of Medicine, University of Colombo, Sri Lanka ${ }^{3}$ \\ Department of Chemistry, Faculty of Sciences, University of Colombo, Sri Lanka ${ }^{4}$
}

DOI: http://doi.org/10.4038/jdp.v11i2.7712

\section{Introduction}

Human epidermal growth factor receptor2 (HER2) protein over expression and/or HER2 gene amplification is linked to dismal outcome of gastric carcinoma (GCa). Immunohistochemistry (IHC) and fluorescence in situ hybridization (FISH) are key methods to identify patients for HER2 targeted therapy. Draw backs of both methods warrant novel tests. The objectives were to determine whether quantitative Polymerase Chain Reaction (qPCR) could serve as a supplementary method to evaluate HER2 status of GCa and investigate the correlation between HER2 assessed by different methods and clinicopathological features.

\section{Methodology}

Twenty GCa patients with known IHC-HER2 scores were evaluated. QPCR was performed for HER2 gene and Amyloid precursor protein (reference gene) in formalin fixed paraffin embedded GCa tissue. Threshold values (Ct) were analyzed using Pfaffl-method to detect HER2 gene amplification.

\section{Results}

HER2 positivity by IHC (protein) and qPCR (gene) were $20 \%$ and $35 \%$ respectively.
Sensitivity and specificity of qPCR was $67 \%$ and $76 \%$ respectively and results were reproducible. HER2 protein positivity was correlated with TNM-stage and Lauren histological types $(\mathrm{P}<0.05)$. Positive expression of HER2 gene correlated with depth of tumour invasion, differentiation and lymph node status $(P<0.05)$. Diagnostic consistency between $\mathrm{IHC}$ and $\mathrm{qPCR}$ $(\mathrm{k}=0.146) \quad$ was slightly agreeable $(0.01<k<0.20)$, having $65 \%$ concordance.

\section{Discussion}

Discrepancies between HER2 positivity by IHC and QPCR were possibly due to transcription activation by other genes in the absence of HER2 gene amplification and the aberrant form of HER2 protein not detected by IHC. Studies also indicate a higher proportion of IHC negative, but HER2 gene amplified GCa by FISH.

\section{Conclusion}

qPCR may be used as a supplementary method for evaluation of HER2 status in gastric carcinoma proven to be HER2 negative by immunohistochemistry. 